\title{
The Traditional Kampo Medicine Tokishakuyakusan Increases Ocular Blood Flow in Healthy Subjects
}

\author{
Shin Takayama, ${ }^{1,2}$ Yukihiro Shiga, ${ }^{3}$ Taiki Kokubun, ${ }^{3}$ Hideyuki Konno, ${ }^{3}$ \\ Noriko Himori, ${ }^{3}$ Morin Ryu, ${ }^{3}$ Takehiro Numata, ${ }^{4}$ Soichiro Kaneko, ${ }^{4}$ \\ Hitoshi Kuroda, ${ }^{5}$ Junichi Tanaka, ${ }^{2}$ Seiki Kanemura, ${ }^{2}$ Tadashi Ishii, ${ }^{2}$ \\ Nobuo Yaegashi, ${ }^{4}$ and Toru Nakazawa ${ }^{3}$ \\ ${ }^{1}$ Comprehensive Education Center for Community Medicine, Tohoku University Graduate School of Medicine, 2-1 Seiryo-Machi, \\ Aoba Ward, Sendai City 980-8575, Japan \\ ${ }^{2}$ Department of Education and Support for Community Medicine, Tohoku University Hospital, 1-1 Seiryo-Machi, Aoba Ward, \\ Sendai City 980-8574, Japan \\ ${ }^{3}$ Department of Ophthalmology, Tohoku University Graduate School of Medicine, 1-1 Seiryo-Machi, Aoba Ward, \\ Sendai City 980-8574, Japan \\ ${ }^{4}$ Department of Obstetrics and Gynecology, Tohoku University Graduate School of Medicine, 1-1 Seiryo-Machi, Aoba Ward, \\ Sendai City 980-8574, Japan \\ ${ }^{5}$ Division of General Medicine, Saitama Medical Center, Jichi Medical University, 1-847 Amanumacho, Omiya Ward, \\ Saitama City 330-8503, Japan
}

Correspondence should be addressed to Shin Takayama; tatahara1492@gmail.com

Received 21 February 2014; Accepted 22 March 2014; Published 24 April 2014

Academic Editor: Wei Jia

Copyright (C) 2014 Shin Takayama et al. This is an open access article distributed under the Creative Commons Attribution License, which permits unrestricted use, distribution, and reproduction in any medium, provided the original work is properly cited.

The aim of this study was to examine the effects of oral administration of kampo medical formulas on ocular blood flow (OBF). A crossover protocol was used to randomly administer five grams of yokukansan, tokishakuyakusan (TSS), keishibukuryogan, or hachimijiogan to 13 healthy blinded subjects (mean age: $37.3 \pm 12.3$ years). The mean blur rate, a quantitative OBF index obtained with laser speckle flowgraphy, was measured at the optic nerve head before and 30 minutes after administration. Blood pressure (BP) and intraocular pressure (IOP) were also recorded. No significant changes were observed in mean BP or IOP after the administration of any of the kampo medical formulas. There was a significant increase in OBF 30 minutes after administration of TSS (100\% to $103.6 \pm 6.9 \%, P<0.01$ ). Next, TSS was administered to 19 healthy subjects (mean age: $32.0 \pm 11.0$ years) and OBF was measured before and 15, 30, 45, and 60 minutes after administration. Plain water was used as a control. OBF increased significantly after TSS administration compared to control $(P<0.01)$ and also increased from 30 to 60 minutes after administration compared to baseline $(P<0.05)$. These results suggest that TSS can increase OBF without affecting BP or IOP in healthy subjects.

\section{Introduction}

Ocular blood flow (OBF) abnormalities have been reported to play an important role in the pathogenesis of many ocular diseases, including glaucoma and diabetic retinopathy $[1-$ 4]. An understanding of these abnormalities is therefore of critical importance in determining the pathophysiological features of these diseases and finding novel treatments.
Japanese kampo medicine has a history spanning more than 1500 years. Kampo medical formulas have been used to treat a variety of physical illnesses and diseases [5], including ocular conditions such as dry eye, blurred vision, decreased visual acuity, and visual field defects [6]. However, the clinical efficacy of kampo medicine has traditionally been evaluated with subjective assessments of symptoms, and there is little quantitative information on the effects of kampo medical 
formulas on the eye [7]. A few such studies have suggested that yokukansan (YKS) has an influence on blood flow in the short posterior ciliary artery in rabbits [8] and that hachimijiogan (HJG) has an effect on blood flow in the central retinal artery in humans [9]. Other studies have reported that tokishakuyakusan (TSS) has an inhibitory effect on platelet aggregation and a relaxative effect on vascular smooth muscle $[10,11]$. Additionally, a study of erythrocyte aggregability and deformability found that keishibukuryogan (KBG) had an influence on microcirculation $[12,13]$. These four kampo medical formulas thus have the potential to influence ocular circulation, but so far, no studies have been conducted to evaluate their effects.

Recently, we have reported that laser speckle flowgraphy (LSFG) and color Doppler imaging can be used to assess the effects of topical medications or acupuncture on OBF, including circulation in the optic nerve head $(\mathrm{ONH})$ and retrobulbar space [14-16]. LSFG allows us to quantify microcirculation in the $\mathrm{ONH}$, the choroid, and the retina simultaneously [17]. It is a noncontact technique based on the laser speckle phenomenon and has been shown to be reliable and reproducible in human eyes, especially in the ONH [18-20]. Furthermore, it can acquire an image of $\mathrm{OBF}$ in just a few seconds [17]. We believe that these characteristics make LSFG a suitable instrument for this study's evaluation of dynamic $\mathrm{OBF}$ alterations in healthy eyes after the administration of kampo formulas.

Thus, the object of this study was to investigate, with healthy volunteers and LSFG, the effects on OBF of kampo formulas (YKS, TSS, KBG, and HJG) traditionally used for the treatment of eye disease.

\section{Methods}

\subsection{Experiment 1 (Ex. 1)}

2.1.1. Subjects. Healthy, nonsmoking volunteers ranging from 20 to 70 years old were recruited from respondents to a poster campaign at Tohoku University Hospital, Miyagi, Japan, between April 2013 and November 2013. Subjects were included in this experiment if baseline intraocular pressure (IOP) was below $22 \mathrm{mmHg}$ in both eyes in a Goldmann applanation tonometry examination, findings were normal in slit lamp and funduscopic examinations, and refractive error was within a range of -8.5 to -0.5 diopters (mean of the included subjects: $-2.2 \pm 2.0$ ). Subjects were excluded if they had a history of ophthalmic or general disorders, had ocular laser or incisional surgery in either eye, or were receiving systemic or topical medication. Subjects abstained from alcohol and caffeine for at least six hours before the measurements. Both eyes of each participant were monitored. The procedures in all experiments followed the tenets of the Declaration of Helsinki and were approved by the committee of Tohoku University Graduate School of Medicine. Written informed consent was obtained from all participants. Overall, 26 eyes of 13 healthy volunteers (mean age, $37.3 \pm 12.3$ years; 6 men and 7 women) were included in this experiment.

2.1.2. Study Design. This was a randomized, double-blinded, crossover experiment.
2.1.3. Randomization. No participants or examiners had any knowledge of kampo medicine and they were blinded to the order of administration of the formulas, which was randomized. The washout period between administrations of the formulas was at least one week. All four formulas were administered to each participant over the course of two months.

2.1.4. Measurement of Clinical Parameters. IOP was determined with Goldmann applanation tonometry. Both systemic blood pressure (BP) and pulse rate (PR) were conventionally measured from the left brachial artery at the height of the heart with an automated BP monitor (HEM-759 E, Omron Corporation, Kyoto, Japan). Mean arterial blood pressure (MBP) was calculated from systolic BP (SBP) and diastolic BP (DBP) according to the following formula: $\mathrm{MBP}=\mathrm{DBP}+0.42(\mathrm{SBP}-\mathrm{DBP})[21-23]$.

2.1.5. LSFG. The principles of LSFG have previously been described in detail $[24,25]$. Briefly, this instrument consists of a fundus camera equipped with a diode laser $(830 \mathrm{~nm}$ wavelength) and an ordinary charge-coupled device sensor $(750 \times$ 360 pixels). This study used the LSFG-NAVI device (Softcare Co., Ltd., Fukutsu, Japan), which has been approved by the Pharmaceuticals and Medical Devices Agency in Japan. Mean blur rate (MBR), a measurement of relative OBF expressed in arbitrary units, is determined using the pattern of speckle contrast produced by the interference of a laser scattered by blood cells moving in the ocular fundus [26]. The MBR images are acquired continuously at the rate of 30 frames per second over a 4-second period. The accompanying analysis software (LSFG Analyzer, version 3.0.43.0) combines all captured images into a composite map of OBF. In this study, we identified the $\mathrm{ONH}$ margin and set this area as the region of interest in the software. The software then automatically calculated the MBR in the ONH from the OBF map, as we have described in a previous report [16].

2.1.6. Intervention. One of the four kampo medical formulas was prepared in $50 \mathrm{~mL}$ of plain hot water and orally administered to the subject. The kampo medical formulas included YKS, TSS, KBG, and HJG. All were produced by Tsumura and Co. (Tokyo, Japan). The compositions of the formulas, which are all mixtures of dried herbs registered in the Pharmacopoeia of Japan, are as follows. YKS contains $4.0 \mathrm{~g}$ Atractylodis Lanceae Rhizoma, $4.0 \mathrm{~g}$ Poria, $3.0 \mathrm{~g}$ Cnidii Rhizoma, 3.0 g Angelicae Radix, 2.0 g Bupleuri Radix, $1.5 \mathrm{~g}$ Glycyrrhizae Radix, and 3.0 g Uncariae Uncis Cum Ramulus; TSS contains $4.0 \mathrm{~g}$ Paeoniae Radix, $4.0 \mathrm{~g}$ Atractylodis Lanceae Rhizoma, 4.0 g Alismatis Rhizoma, 4.0 g Poria, 3.0 g Cnidii Rhizome, and 3.0 g Angelicae Radix; KBG contains 3.0 g Cinnamomi Cortex, 3.0 g Paeoniae Radix, 3.0 g Persicae Semen, $3.0 \mathrm{~g}$ Poria, and $3.0 \mathrm{~g}$ Moutan bark; and HJG contains $6.0 \mathrm{~g}$ Rehmanniae Radix, $3.0 \mathrm{~g}$ Corni Fructus, $3.0 \mathrm{~g}$ Dioscoreae Rhizoma, $3.0 \mathrm{~g}$ Alismatis Rhizoma, $3.0 \mathrm{~g}$ Poria, $2.5 \mathrm{~g}$ Moutan Cortex, $1.0 \mathrm{~g}$ Cinnamomi Cortex, and $0.5 \mathrm{~g}$ Aconiti Tuber.

2.1.7. Testing Protocol. All tests were performed between 6:00 and 8:00 p.m. at an ambient room temperature of 25 degrees 
TABLE 1: Baseline variables in the four study groups (Experiment 1).

\begin{tabular}{lllllc}
\hline Variable & YKS & TSS & KBG & HJG & $P$ value \\
\hline OBF $(\mathrm{AU})$ & $24.5 \pm 3.5$ & $22.8 \pm 2.8$ & $23.4 \pm 3.8$ & $23.6 \pm 3.8$ \\
IOP $(\mathrm{mmHg})$ & $12.9 \pm 2.5$ & $14.2 \pm 2.4$ & $13.6 \pm 2.5$ & $13.2 \pm 2.7$ \\
SBP (mmHg) & $109.7 \pm 10.9$ & $113.3 \pm 10.9$ & $111.8 \pm 9.2$ & $112.0 \pm 14.4$ \\
DBP (mmHg) & $64.8 \pm 12.0$ & $66.6 \pm 9.9$ & $67.3 \pm 10.3$ & $64.4 \pm 7.6$ \\
MBP (mmHg) & $83.7 \pm 10.7$ & $85.4 \pm 10.0$ & $86.0 \pm 9.0$ & $84.4 \pm 9.8$ \\
PR (beats/min) & $73.6 \pm 7.7$ & $75.5 \pm 10.7$ & $71.0 \pm 8.2$ & $77.2 \pm 6.8$ & 0.95 \\
\hline
\end{tabular}

Data are expressed as mean \pm standard deviation. Differences between groups were assessed with the Kruskal-Wallis test.

Celsius. On the day of the test, after a slit lamp examination, 0.4\% tropicamide (Mydrin M; Santen Pharmaceutical Co., Ltd., Osaka, Japan) was used to dilate the pupil. All subjects received a funduscopic examination, and IOP was measured with Goldmann applanation tonometry. To ensure that systemic hemodynamic conditions in each subject were consistent, all subjects rested in a sitting position for 10 minutes before the start of the investigation, in accordance with previous studies [16-20]. Immediately after the 10-minute rest period, systemic BP, PR, and IOP were recorded, and triplicate measurements of OBF were made with LSFG. The formula was administered, and after 30 minutes, triplicate OBF measurements were made again. The entire procedure took place in a darkened room. Normalized MBR was used for the statistical analysis.

2.1.8. Statistical Analysis. The analysis used percentage values (\%) calculated by defining the baseline measurement variables as $100 \%$. The values were the mean \pm standard deviation. Measurements of baseline variables made on different days were compared with the Kruskal-Wallis test. The Wilcoxon signed-rank test was used to assess differences in the values before and after kampo administration. All statistical analyses were performed with JMP software (Pro version 10.0.2; SAS Institute Japan, Inc., Tokyo, Japan). The difference was considered significant when the $P$ value was less than 0.05 .

\subsection{Experiment 2 (Ex. 2)}

2.2.1. Subjects. The inclusion criteria, recruitment of subjects, and procedures of the test were the same as those in Ex. 1. Overall, 38 eyes of 19 healthy volunteers (mean age: $32.0 \pm 11.0$ years, male : female $=8: 11$ ) were included in this experiment.

2.2.2. Study Design. This was a double-blinded, controlled experiment.

2.2.3. Measurement of Clinical Parameters. Measurement of IOP, BP, PR, and OBF was the same as in Ex. 1.

2.2.4. Intervention. Five grams of TSS prepared in $50 \mathrm{~mL}$ of hot water was administered to each subject. As a control, $50 \mathrm{~mL}$ of plain hot water was also administered to each subject on a different day, with a washout period of at least one week between the administration of TSS and water.
2.2.5. Kampo Diagnostic Questionnaire. A questionnaire was given to the subjects to determine their scores for "qi," "blood," and "fluid," traditional diagnostic criteria used in kampo medicine. The questionnaire was obtained from a kampo manual written by Terasawa [27]. "Qi," "blood," and "fluid" are considered fundamental components of the human body in kampo medicine, and disorders and disease are associated with imbalances of these components. These imbalances are classified as "qi deficiency," "qi stagnation," "qi counter flow," "blood deficiency," "blood stasis," and "fluid retention." [28].

2.2.6. Testing Protocol. The procedures of the test were the same as in Ex. 1. Systemic BP, PR, and OBF were measured just before and 15, 30, 45, and 60 minutes after administration of TSS or water. IOP was recorded before and 60 minutes after administration.

2.2.7. Statistical Analysis. The determination of baseline values was with the same method as in Ex. 1. LSFG values after administration of TSS and water were compared at each time point with a two-way analysis of variance (ANOVA). The repeated ANOVA values and post hoc Dunnett's test were used for a statistical comparison of baseline and postadministration values. Differences were considered significant with a $P$ value of less than 0.05 .

\section{Results}

\subsection{Ex. 1}

3.1.1. Comparison of Baseline Variables of the Subjects. Table 1 summarizes the measurements of the baseline variables in the subjects before the administration of each formula. There were no significant differences in OBF, IOP, SBP, DBP, MBP, or $\mathrm{PR}$ between the subjects before they were administered any of the formulas.

3.1.2. Alteration of Systemic Variables, IOP, and OBF after Administration of Each Kampo Formula. No significant changes were observed in IOP or MBP after the administration of any of the kampo formulas. PR significantly decreased after the administration of YKS, TSS, and HJG (Table 2). Thirty minutes after the administration of TSS, LSFG measurements of OBF revealed significant increases (100\% to $103.6 \pm 6.9 \%$, $P<0.01)$. By contrast, 30 minutes after the administration 
TABLE 2: Alteration of IOP and systemic variables in response to administration of each kampo formula (Experiment 1).

\begin{tabular}{|c|c|c|c|c|}
\hline & Variable & Baseline & 30 minutes after treatment & $P$ value \\
\hline \multirow{4}{*}{ YKS } & OBF change (\%) & $100.0 \pm 0.0$ & $96.9 \pm 6.7$ & 0.05 \\
\hline & $\mathrm{IOP}(\mathrm{mmHg})$ & $12.9 \pm 2.5$ & $13.0 \pm 2.9$ & 0.73 \\
\hline & $\mathrm{MBP}(\mathrm{mmHg})$ & $83.7 \pm 10.7$ & $87.8 \pm 8.2$ & 0.13 \\
\hline & PR (beats/min) & $73.6 \pm 7.7$ & $66.9 \pm 7.8$ & $<0.01$ \\
\hline \multirow{4}{*}{ TSS } & OBF change (\%) & $100.0 \pm 0.0$ & $103.6 \pm 6.9$ & $<0.01$ \\
\hline & IOP (mmHg) & $14.2 \pm 2.4$ & $14.1 \pm 2.5$ & 0.98 \\
\hline & MBP (mmHg) & $85.4 \pm 10.0$ & $86.8 \pm 4.9$ & 0.27 \\
\hline & PR (beats/min) & $75.5 \pm 10.7$ & $67.0 \pm 8.0$ & $<0.01$ \\
\hline \multirow{4}{*}{ KBG } & OBF change (\%) & $100.0 \pm 0.0$ & $101.8 \pm 6.9$ & 0.35 \\
\hline & $\mathrm{IOP}(\mathrm{mmHg})$ & $13.6 \pm 2.5$ & $13.3 \pm 2.9$ & 0.17 \\
\hline & $\mathrm{MBP}(\mathrm{mmHg})$ & $86.0 \pm 9.0$ & $86.9 \pm 8.9$ & 0.74 \\
\hline & PR (beats/min) & $71.0 \pm 8.2$ & $66.6 \pm 7.9$ & 0.05 \\
\hline \multirow{4}{*}{ HJG } & OBF changc (\%) & $100.0 \pm 0.0$ & $100.0 \pm 6.9$ & 0.89 \\
\hline & $\mathrm{IOP}(\mathrm{mmHg})$ & $13.2 \pm 2.7$ & $13.1 \pm 2.5$ & 0.81 \\
\hline & MBP (mmHg) & $84.4 \pm 9.8$ & $85.9 \pm 6.0$ & 0.74 \\
\hline & PR (beats/min) & $77.2 \pm 6.8$ & $67.2 \pm 7.7$ & $<0.01$ \\
\hline
\end{tabular}

Data are expressed as mean \pm standard deviation. The Wilcoxon signed-rank test was applied to assess differences in the values before and after kampo administration.

of YKS, KBG, or HJG there were no significant changes in OBF. Additionally, there were no adverse events from administration of any of the kampo medical formulas during the experiment.

\subsection{Ex. 2}

3.2.1. Comparison of Baseline Variables in the Subjects. Table 3 summarizes the baseline variables of the subjects before administration of water or TSS. There were no significant differences in OBF, SBP, DBP, MBP, or PR, before the administration of either substance, although there was a small, though statistically significant, difference in IOP.

3.2.2. Alteration in $\mathrm{OBF}$ after Administration. OBF after the administration of TSS or water was significantly different $(P<0.01)$ (Table 4). OBF increased significantly 30, 45, and 60 minutes after administration of TSS (100\% to $104.1 \pm 5.3 \%$; $P<0.05,104.2 \pm 4.8 \% ; P<0.05,104.0 \pm 5.5 \% ; P<0.05)$ (Figure 1). According to the results of the kampo diagnostic questionnaire, 5 subjects had "qi deficiency," 3 subjects had "qi stagnation," 6 subjects had "qi counter flow," 4 subjects had "blood deficiency," 10 subjects had "blood stasis," and 8 subjects had "fluid retention." OBF increased significantly 15 to 60 minutes after the administration of TSS in the group of subjects with "blood deficiency" and "blood stasis" (15 minutes, $P<0.05$; 30 minutes, $P<0.05$; 45 minutes, $P<0.05 ; 60$ minutes, $P<0.05)$ and in the group with "blood deficiency" and "fluid retention" (15 minutes, $P<0.05$; 30 minutes, $P<0.05$; 45 minutes, $P<0.05$; 60 minutes, $P<0.05)$ (Figures 2(a) and 2(b)). Figure 3 shows a representative image of OBF in the ONH before administration of TSS, as well as 30 and 60 minutes after administration.
TABLE 3: Baseline variables in the 2 study groups (Experiment 2).

\begin{tabular}{llll}
\hline Variable & Control & TSS & $P$ value \\
\hline OBF (AU) & $25.1 \pm 4.3$ & $24.7 \pm 4.6$ & 0.25 \\
IOP (mmHg) & $12.8 \pm 2.2$ & $13.8 \pm 2.0$ & $<0.01$ \\
SBP (mmHg) & $106.2 \pm 11.4$ & $110.1 \pm 14.2$ & 0.16 \\
DBP (mmHg) & $64.9 \pm 8.4$ & $67.9 \pm 10.8$ & 0.09 \\
MBP (mmHg) & $82.3 \pm 9.1$ & $85.7 \pm 11.7$ & 0.11 \\
PR (beats/min) & $73.1 \pm 13.1$ & $73.2 \pm 9.5$ & 0.65 \\
\hline
\end{tabular}

Data are expressed as mean \pm standard deviation. Differences between groups were assessed with the Kruskal-Wallis test.

\section{Discussion}

Ex. 1 revealed that, of four candidate kampo formulas, only TSS had the effect of increasing OBF. This was somewhat in contrast to previous studies, which have found that YKS has an influence on blood flow in the short posterior ciliary artery in rabbits [8] and that HJG has an effect on blood flow in the central retinal artery in humans [9]. This may be due to differences in OBF measurement technique and location between these previous studies and the present one. The previous studies evaluated OBF from the retrobulbar side, whereas this study evaluated OBF from the ocular side, in the ONH.

The mechanism of the effects of TSS and KBG on circulation has been described in several reports. Nasu et al. reported that TSS had an inhibitory effect on platelet aggregation [10]. $\mathrm{KBG}$ has been reported to improve endothelial function in patients with metabolic syndrome [29]. Additionally, an evaluation of erythrocyte aggregability and deformability in patients with multiple old lacunar infarctions found that KBG improved microcirculation $[12,13]$. TSS has a number of 
TABLE 4: Alteration of ocular blood flow and clinical variables in response to administration of kampo formula (Experiment 2).

\begin{tabular}{|c|c|c|c|c|c|c|c|}
\hline & Variable & Baseline & $\begin{array}{l}15 \text { minutes } \\
\text { after treatment }\end{array}$ & $\begin{array}{l}30 \text { minutes } \\
\text { after treatment }\end{array}$ & $\begin{array}{l}45 \text { minutes } \\
\text { after treatment }\end{array}$ & $\begin{array}{l}60 \text { minutes } \\
\text { after treatment }\end{array}$ & $P$ value \\
\hline \multirow{6}{*}{ Control } & OBF change (\%) & $100.0 \pm 0.0$ & $99.3 \pm 8.0$ & $99.8 \pm 8.1$ & $101.7 \pm 7.6$ & $101.4 \pm 8.1$ & 0.49 \\
\hline & IOP (mmHg) & $12.8 \pm 2.2$ & & & & $12.4 \pm 2.1$ & 0.11 \\
\hline & $\mathrm{SBP}(\mathrm{mmHg})$ & $106.2 \pm 11.4$ & $106.9 \pm 11.5$ & $106.2 \pm 10.2$ & $108.0 \pm 13.0$ & $108.1 \pm 13.5$ & 0.98 \\
\hline & $\mathrm{DBP}(\mathrm{mmHg})$ & $64.9 \pm 8.4$ & $67.5 \pm 12.0$ & $65.3 \pm 7.4$ & $65.8 \pm 7.4$ & $66.9 \pm 8.4$ & 0.90 \\
\hline & $\mathrm{MBP}(\mathrm{mmHg})$ & $82.3 \pm 9.1$ & $84.1 \pm 11.0$ & $82.5 \pm 8.3$ & $83.5 \pm 9.0$ & $84.2 \pm 10.0$ & 0.96 \\
\hline & $\mathrm{PR}$ (beats/min) & $73.1 \pm 13.1$ & $66.7 \pm 8.1$ & $65.7 \pm 7.5$ & $67.7 \pm 7.8$ & $66.3 \pm 8.1$ & 0.12 \\
\hline \multirow{6}{*}{ TSS } & OBF change (\%) & $100.0 \pm 0.0$ & $102.7 \pm 7.0$ & $104.1 \pm 5.3$ & $104.2 \pm 4.8$ & $104.0 \pm 5.5$ & $<0.01$ \\
\hline & IOP (mmHg) & $13.8 \pm 2.0$ & & & & $13.6 \pm 1.8$ & 0.41 \\
\hline & $\mathrm{SBP}(\mathrm{mmHg})$ & $110.1 \pm 14.2$ & $110.1 \pm 10.0$ & $108.6 \pm 10.3$ & $108.3 \pm 11.5$ & $109.0 \pm 10.7$ & 0.98 \\
\hline & $\mathrm{DBP}(\mathrm{mmHg})$ & $67.9 \pm 10.8$ & $69.3 \pm 9.8$ & $68.3 \pm 8.4$ & $69.3 \pm 9.2$ & $71.0 \pm 10.8$ & 0.90 \\
\hline & MBP (mmHg) & $85.7 \pm 11.7$ & $86.4 \pm 9.1$ & $85.2 \pm 8.5$ & $85.7 \pm 9.1$ & $87.0 \pm 10.1$ & 0.45 \\
\hline & PR (beats/min) & $67.9 \pm 10.8$ & $69.3 \pm 9.8$ & $68.3 \pm 8.4$ & $69.3 \pm 9.2$ & $71.0 \pm 10.8$ & 0.19 \\
\hline
\end{tabular}

Data are expressed as mean \pm standard deviation. The Wilcoxon signed-rank test was applied to assess differences in IOP before and after kampo administration. A one-way analysis of variance was used to assess differences in other values before and after kampo administration.

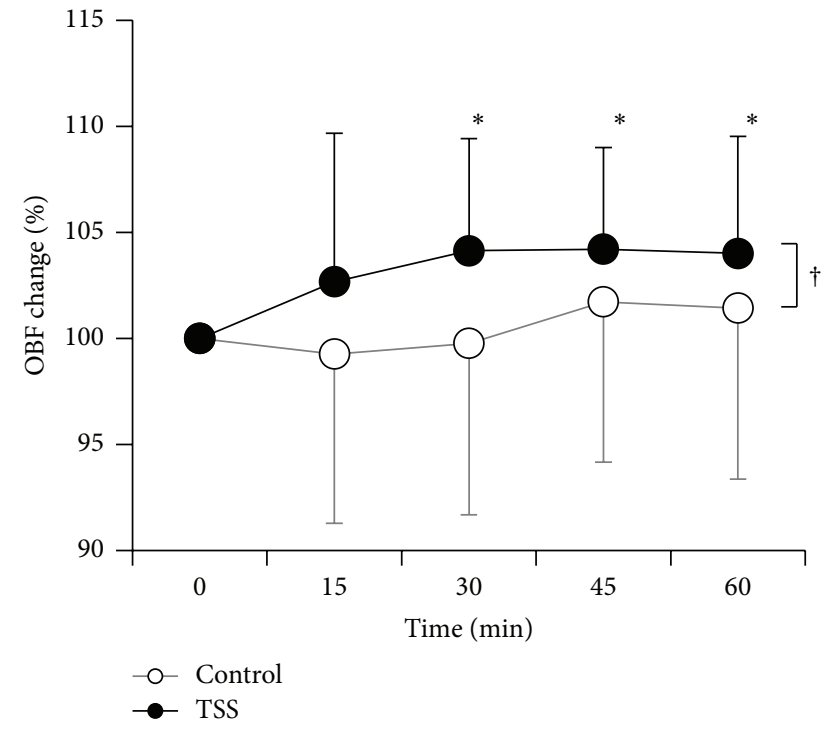

FIGURE 1: Dynamic OBF changes in response to administration of TSS $(\bullet)$ and control $(O)$. Data are expressed as mean \pm standard deviation. The dagger indicates a statistically significant difference between TSS and control (two-way analysis of variance; ANOVA). The asterisk indicates a statistically significant difference from the baseline (repeated measurements of ANOVA, with post hoc Dunnett's test).

components in common with YKS, KBG, and HJG. Atractylodis Lanceae Rhizoma, included in YKS and TSS, has been reported to inhibit platelet aggregation [10]. Poria, included in YKS, TSS, KBG, and $\mathrm{HJG}$, has been reported to have a similar effect [10]. Paeoniae Radix, included in TSS and $\mathrm{KBG}$, has been reported to relax vascular smooth muscle [11]. Polyphenols of Cinnamomi Cortex or cinnamaldehyde, included in KBG and HJG, have been reported to have an endothelium-dependent relaxative effect [30-33]. Moreover, these four kampo formulas include multiple physiologically active substances that have yet to be fully identified. The interactions between them are also not yet fully understood.

In Ex. 2, we found that TSS increased OBF without affecting BP or IOP. This additional experiment supported the preliminary results of Ex. 1. In kampo practice, TSS is used to treat "blood deficiency," "fluid retention," and "blood stasis" [34], which are part of six conditions used in kampo practice and described by Terasawa. These conditions also include "qi deficiency," "qi stagnation," and "qi counter flow." Classification of patients depends on their symptoms and a physical examination [27, 28, 34]. "Blood deficiency" and "blood stasis" are believed to be associated with general symptoms of eye disease such as blurred vision, decreased visual acuity, and visual field defects, while increased IOP is believed to be associated with "fluid retention" and "qi stagnation." Kampo formulas are chosen based on the patient's symptoms and the results of a physical examination in a clinical setting, a methodology that has been in use since ancient times. Patients with combined "blood deficiency" and "blood stasis" or combined "blood deficiency" and "fluid retention" are considered suitable cases for treatment with TSS in kampo practice, and in Ex. 2 of this study, the OBF increase was in fact most remarkable in subjects with these conditions. This result supports the existence of a relationship between the effectiveness of kampo formulas in practice and diagnoses made according to kampo theory. The effectiveness of the formulas has traditionally been judged only by subjectively measured improvements, with quantitative and objective reports of the effects of kampo formulas on ocular circulation being very limited. The present study sought to alleviate this deficiency. We believe our results indicate that, to be most effective, kampo formulas should be chosen after patients are evaluated by both traditional kampo diagnostic practices and western medical techniques.

The authors have previously reported that 60 minutes after administration of topical tafluprost, LSFG measurements of OBF increased significantly in the eyes of normal subjects and patients with normal tension glaucoma (NTG) 


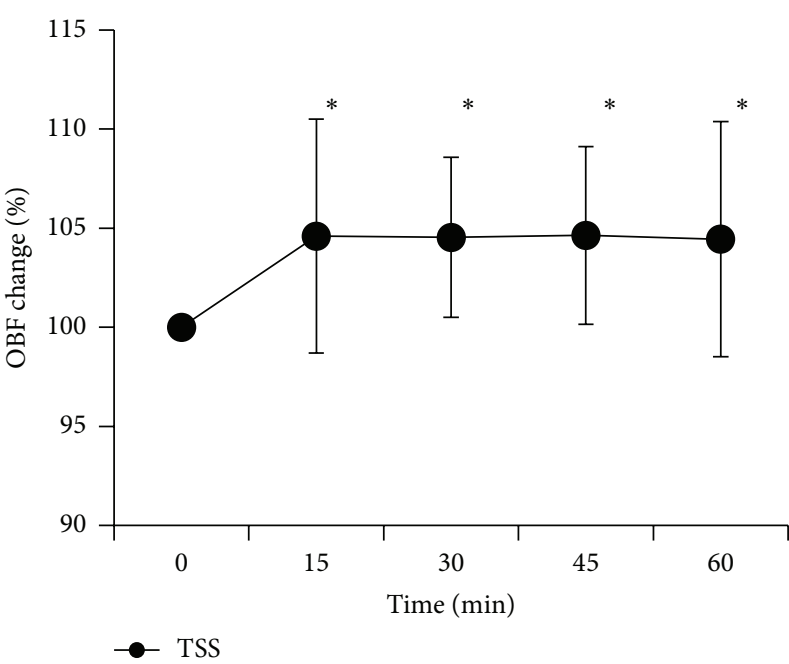

(a)

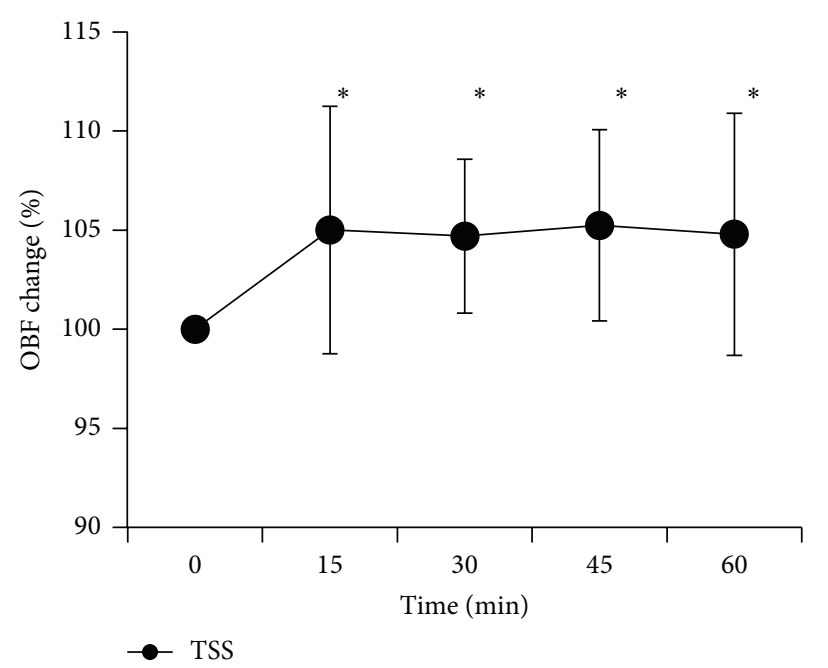

(b)

FIGURE 2: Dynamic OBF changes in subjects with blood deficiency and blood stasis (a) and with blood deficiency and fluid retention (b) in response to administration of TSS. Data are expressed as mean \pm standard deviation. The asterisk indicates a statistically significant difference from the baseline (repeated measurements of ANOVA, with post hoc Dunnett's test).

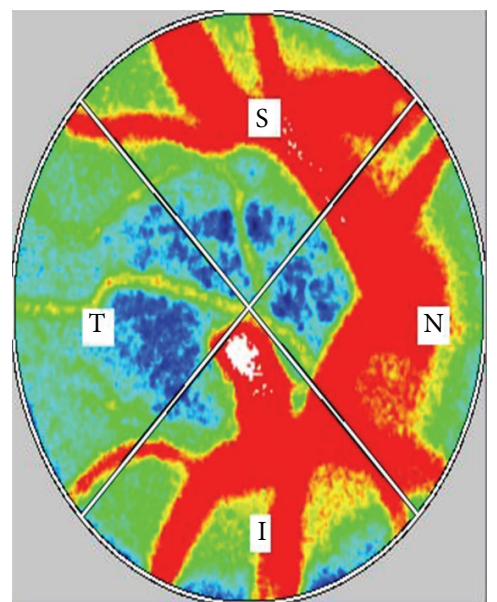

(a)

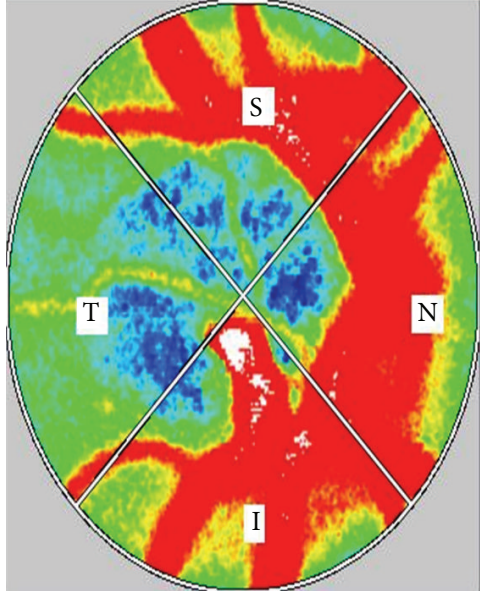

(b)

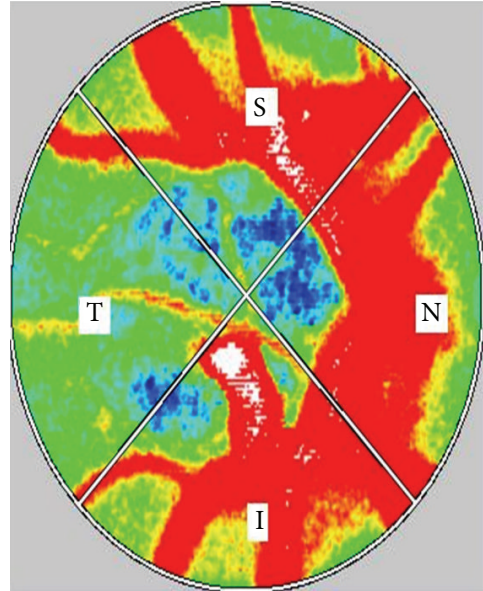

(c)

FIGURE 3: Representative MBR images of the entire ONH before administration and 30 and 60 minutes after the administration of TSS in healthy subjects (right eye). (a) Composite blood flow map of the ONH before the administration of TSS. The MBR value is 21.2. (b) Composite blood flow map of the ONH 30 minutes after the administration of TSS. The MBR value is 24.8. (c) Composite blood flow map of the ONH 60 minutes after the administration of TSS. The MBR value is 26.1.

(100\% to $104.3 \pm 6.6 \%, P<0.01)$ [16]. This study revealed that TSS had a similar ability to significantly increase OBF in a similar timeframe. In addition, while tafluprost led to a significant decrease in IOP, TSS had no effect on IOP. Recently, it has been suggested that impaired ocular circulation may contribute to the progression of glaucomatous damage $[35,36]$. New drugs or interventions to improve ocular hemodynamics have thus become a promising target of research. Hypotension often coincides with NTG [37], and as oral vasodilators are difficult to use, to both improve OBF and treat hypotension, TSS administration, alone and in combination with topical medications such as tafluprost, may be an effective strategy to improve fundus circulation in glaucoma patients, especially those with NTG.

Limitations. We found that PR decreased after the administration of YKS, TSS, KBG, and HJG. Moreover, this decrease occurred in all groups of subjects including those who were administered a control substance, leading us to believe that it was a nonspecific response. The subjects waited in a quiet, darkened room for over 30 minutes, and the observed decrease in PR may have been an effect of relaxation. The other limitation of this study was the small number of healthy subjects included and the short observation time. In the 
future, we hope to conduct a longitudinal clinical study to determine the effectiveness of TSS in patients with eye disease, especially NTG.

\section{Conclusions}

TSS increased OBF without affecting BP or IOP in healthy subjects. Further study will be needed to investigate the effect of TSS in patients with eye disease.

\author{
Abbreviations \\ OBF: Ocular blood flow \\ YKS: Yokukansan \\ TSS: Tokishakuyakusan \\ KBG: Keishibukuryogan \\ HJG: Hachimijiogan \\ LSFG: Laser speckle flowgraphy \\ ONH: Optic nerve head \\ Ex.: Experiment \\ IOP: Intraocular pressure \\ BP: $\quad$ Blood pressure \\ PR: Pulse rate \\ MBP: Mean arterial blood pressure \\ SBP: Systolic BP \\ DBP: Diastolic BP \\ MBR: Mean blur rate \\ NTG: Normal tension glaucoma.
}

\section{Conflict of Interests}

The authors declare that they have no conflict of interests.

\section{Authors' Contribution}

Shin Takayama and Yukihiro Shiga equally contributed to this work. Shin Takayama took part in planning the study, randomized the order of administration of the kampo medical formulas, and wrote the paper. Yukihiro Shiga took part in planning the study, performed the measurements, and wrote the paper. Taiki Kokubun, Hideyuki Konno, Noriko Himori, and Morin Ryu took part in planning the study and performed the measurements. Takehiro Numata performed the statistical analysis. Soichiro Kaneko took part in planning the study and provided advice for the statistical analysis. Hitoshi Kuroda, Junichi Tanaka, Seiki Kanemura, Tadashi Ishii, and Toru Nakazawa provided advice for the protocol and the paper. Tadashi Ishii, Nobuo Yaegashi, and Toru Nakazawa were responsible for the study design and execution and assisted in writing the paper.

\section{Acknowledgments}

The authors thank Seri Takahashi, Shiori Suzuki, and Haruka Sato for technical support and thank Tim Hilts for reviewing the paper. Tohoku University Graduate School of Medicine did receive a grant from Tsumura, a Japanese manufacturer of kampo medicine.

\section{References}

[1] F. Berisha, G. T. Feke, T. Hirose, J. W. McMeel, and L. R. Pasquale, "Retinal blood flow and nerve fiber layer measurements in early-stage open-angle glaucoma," American Journal of Ophthalmology, vol. 146, no. 3, pp. 466-472, 2008.

[2] J. E. Grunwald, A. J. Brucker, S. E. Grunwald, and C. E. Riva, "Retinal hemodynamics in proliferative diabetic retinopathy: a laser Doppler velocimetry study," Investigative Ophthalmology \& Visual Science, vol. 34, no. 1, pp. 66-71, 1993.

[3] E. M. Kohner, "Dynamic changes in the microcirculation of diabetics as related to diabetic microangiopathy," Acta Medica Scandinavica, Supplement, vol. 578, pp. 41-47, 1975.

[4] J. E. Grunwald, C. E. Riva, and R. A. Stone, "Retinal autoregulation in open-angle glaucoma," Ophthalmology, vol. 91, no. 12, pp. $1690-1694,1984$.

[5] Y. Sato, Introduction to Kampo, The Japan Society for Oriental Medicine, Elsevier, pp. 6-8, 2005.

[6] Y. Sato, Introduction to Kampo, The Japan Society for Oriental Medicine, Elsevier, pp. 165-168, 2005.

[7] S. Hayasaka, T. Kodama, and A. Ohira, "Traditional Japanese herbal (kampo) medicines and treatment of ocular diseases: a review," The American Journal of Chinese Medicine, vol. 40, no. 5, pp. 887-904, 2012.

[8] R. Yamada, "Hemodynamic examination on short posterior ciliary artery of the rabbit after the administration of yokukansan," Kampo and the Newest Therapy, vol. 20, no. 2, pp. 175-178, 2011.

[9] H. Isobe, K. Yamamoto, and J.-C. Cyong, "Effects of Hachimijio-gan (Ba-wei-di-huang-wan) on blood flow in the human central retinal artery," The American Journal of Chinese Medicine, vol. 31, no. 3, pp. 425-435, 2003.

[10] Y. Nasu, M. Iwashita, M. Saito, S. Fushiya, and N. Nakahata, "Inhibitory effects of Atractylodis Lanceae Rhizoma and Poria on collagenor thromboxane A2-induced aggregation in rabbit platelets," Biological and Pharmaceutical Bulletin, vol. 32, no. 5, pp. 856-860, 2009.

[11] H. Goto, Y. Shimada, Y. Akechi, K. Kohta, M. Hattori, and K. Terasawa, "Endothelium-dependent vasodilator effect of extract prepared from the roots of Paeonia lactiflora on isolated rat aorta," Planta Medica, vol. 62, no. 5, pp. 436-439, 1996.

[12] T. Itoh, K. Terasawa, K. Kohta, N. Shibahara, H. Tosa, and Y. Hiyama, "Effects of Keishi-bukuryo-gan and trapidil on the microcirculation in patients with cerebro-spinal vascular disease," Journal of Medical and Pharmaceutical Society For WAKAN-YAKU, vol. 9, no. 1, pp. 40-46, 1992.

[13] H. Hikiami, H. Goto, N. Sekiya et al., "Comparative efficacy of Keishi-bukuryo-gan and pentoxifylline on RBC deformability in patients with "oketsu" syndrome," Phytomedicine, vol. 10, no. 6-7, pp. 459-466, 2003.

[14] S. Takayama, M. Watanabe, H. Kusuyama et al., "Evaluation of the effects of acupuncture on blood flow in humans with ultrasound color Doppler imaging," Evidence-Based Complementary and Alternative Medicine, vol. 2012, Article ID 513638, 8 pages, 2012.

[15] T. Seki, S. Takayama, T. Nakazawa et al., "Short-term effects of acupuncture on open-angle glaucoma in retrobulbar circulation: additional therapy to standard medication," Evidencebased Complementary and Alternative Medicine, vol. 2011, Article ID 157090, 6 pages, 2011.

[16] S. Tsuda, Y. Yokoyama, N. Chiba et al., "Effect of topical tafluprost on optic nerve head blood flow in patients with myopic disc type," Journal of Glaucoma, vol. 22, no. 5, pp. 398-403, 2013. 
[17] T. Sugiyama, M. Araie, C. E. Riva, L. Schmetterer, and S. Orgul, "Use of laser speckle flowgraphy in ocular blood flow research," Acta Ophthalmologica, vol. 88, no. 7, pp. 723-729, 2010.

[18] N. Aizawa, Y. Yokoyama, N. Chiba et al., "Reproducibility of retinal circulation measurements obtained using laser speckle flowgraphy-NAVI in patients with glaucoma," Clinical Ophthalmology, vol. 5, no. 1, pp. 1171-1176, 2011.

[19] Y. Shiga, M. Shimura, T. Asano et al., "The influence of posture change on ocular blood flow in normal subjects, measured by laser speckle flowgraphy," Current Eye Research, vol. 38, no. 6, pp. 691-698, 2013.

[20] Y. Shiga, K. Omodaka, H. Kunikata et al., "Waveform analysis of ocular blood flow and the early detection of normal tension glaucoma," Investigative Ophthalmology \& Visual Science, vol. 54, no. 12, pp. 7699-7706, 2013.

[21] F. N. Sayegh and E. Weigelin, "Functional ophthalmodynamometry: comparison between brachial and ophthalmic blood pressure in sitting and supine position," Angiology, vol. 34, no. 3, pp. 176-182, 1983.

[22] A. Longo, M. H. Geiser, and C. E. Riva, "Posture changes and subfoveal choroidal blood flow," Investigative Ophthalmology \& Visual Science, vol. 45, no. 2, pp. 546-551, 2004.

[23] P. Kaeser, S. Orgül, C. Zawinka, G. Reinhard, and J. Flammer, "Influence of change in body position on choroidal blood flow in normal subjects," The British Journal of Ophthalmology, vol. 89, no. 10, pp. 1302-1305, 2005.

[24] Y. Tamaki, M. Araie, K. Tomita, M. Nagahara, A. Tomidokoro, and H. Fujii, "Non-contact, two-dimensional measurement of tissue circulation in choroid and optic nerve head using laser speckle phenomenon," Experimental Eye Research, vol. 60, no. 4, pp. 373-383, 1995.

[25] H. Isono, S. Kishi, Y. Kimura, N. Hagiwara, N. Konishi, and H. Fujii, "Observation of choroidal circulation using index of erythrocytic velocity," Archives of Ophthalmology, vol. 121, no. 2, pp. 225-231, 2003.

[26] N. Konishi, Y. Tokimoto, K. Kohra, and H. Fujii, "New laser speckle flowgraphy system using CCD camera," Optical Review, vol. 9, no. 4, pp. 163-169, 2002.

[27] K. Terasawa, Shoreikaramanabu Wakanshinryogaku, Igakushoin, Tokyo, Japan, 3rd edition, 2011.

[28] M. Arai, K. Arai, C. Hioki et al., "Evaluation of medical students using the, "qi, blood, and fluid" system of Kampo medicine," The Tokai Journal of Experimental and Clinical Medicine, vol. 38, no. 1, pp. 37-41, 2013.

[29] Y. Nagata, H. Goto, H. Hikiami et al., "Effect of keishibukuryogan on endothelial function in patients with at least one component of the diagnostic criteria for metabolic syndrome: a controlled clinical trial with crossover design," Evidence-Based Complementary and Alternative Medicine, vol. 2012, Article ID 359282, 10 pages, 2012.

[30] L. Gongwang, Fundamentals of Formulas of Chinese Medicine, Hua Xia, 2002.

[31] K. Tanikawa, H. Goto, N. Nakamura et al., "Endotheliumdependent vasodilator effect of tannin extract from Cinnamonomi Cortex on isolated rat aorta," Journal of Medical and Pharmaceutical Society for WAKAN-YAKU, vol. 16, pp. 45-50, 1999.

[32] H. Goto, Y. Shimada, Y. Akechi, K. Kohta, M. Hattori, and K. Terasawa, "Endothelium-dependent vasodilator effect of extract prepared from the roots of Paeonia lactiflora on isolated rat aorta," Planta Medica, vol. 62, no. 5, pp. 436-439, 1996.
[33] A. Yanaga, H. Goto, T. Nakagawa, H. Hikiami, N. Shibahara, and Y. Shimada, "Cinnamaldehyde induces endotheliumdependent and -independent vasorelaxant action on isolated rat aorta," Biological and Pharmaceutical Bulletin, vol. 29, no. 12, pp. 2415-2418, 2006.

[34] Y.-L. Xue, H.-X. Shi, F. Murad, and K. Bian, "Vasodilatory effects of cinnamaldehyde and its mechanism of action in the rat aorta," Vascular Health and Risk Management, vol. 7, pp. 273-280, 2011.

[35] M. Satilmis, S. Orgül, B. Doubler, and J. Flammer, "Rate of progression of glaucoma correlates with retrobulbar circulation and intraocular pressure," American Journal of Ophthalmology, vol. 135, no. 5, pp. 664-669, 2003.

[36] J. Schumann, S. Orgül, K. Gugleta, B. Dubler, and J. Flammer, "Interocular difference in progression of glaucoma correlates with interocular differences in retrobulbar circulation," American Journal of Ophthalmology, vol. 129, no. 6, pp. 728-733, 2000.

[37] V. P. Costa, E. S. Arcieri, and A. Harris, "Blood pressure and glaucoma," British Journal of Ophthalmology, vol. 93, no. 10, pp. 1276-1282, 2009. 


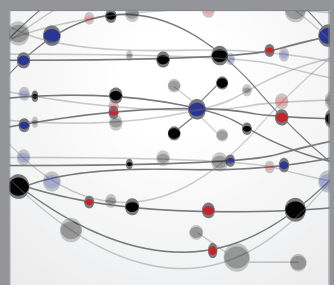

The Scientific World Journal
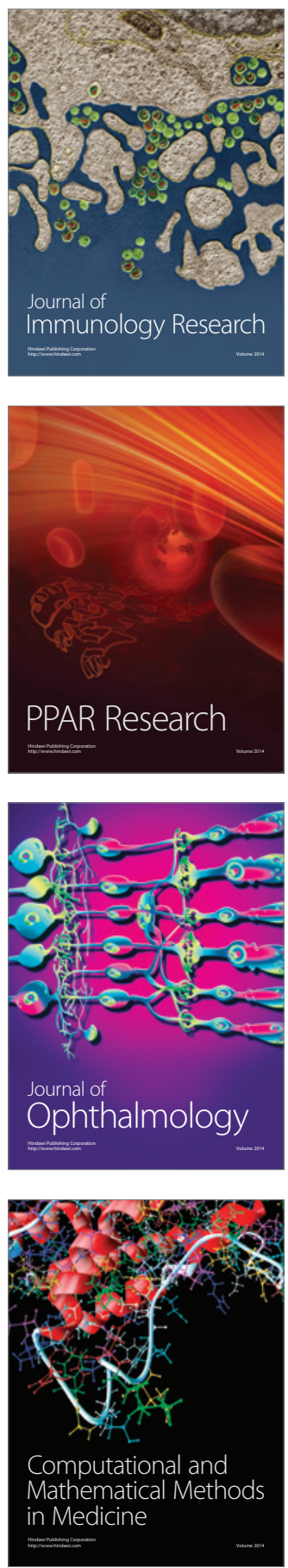

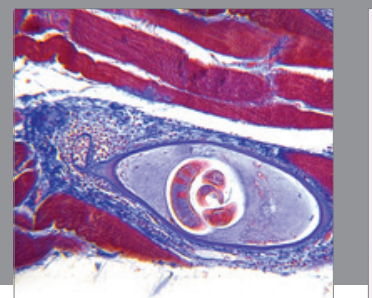

Gastroenterology

Research and Practice
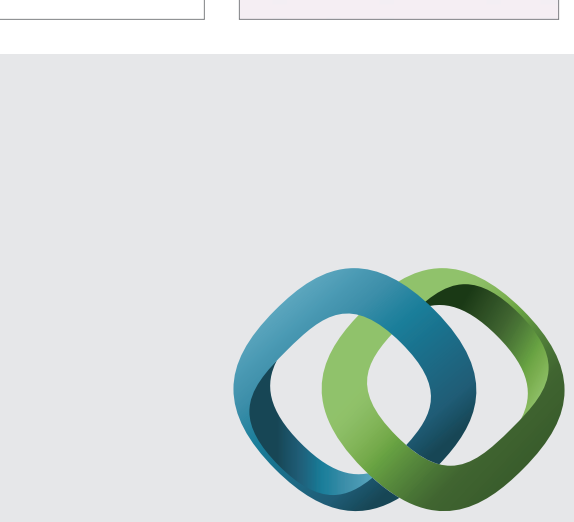

\section{Hindawi}

Submit your manuscripts at

http://www.hindawi.com
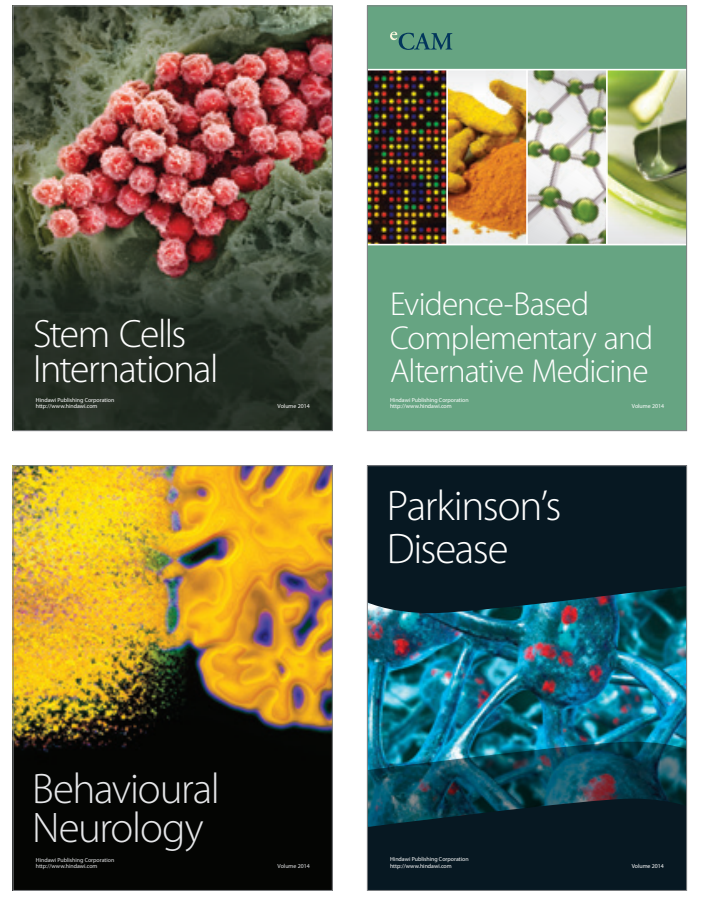
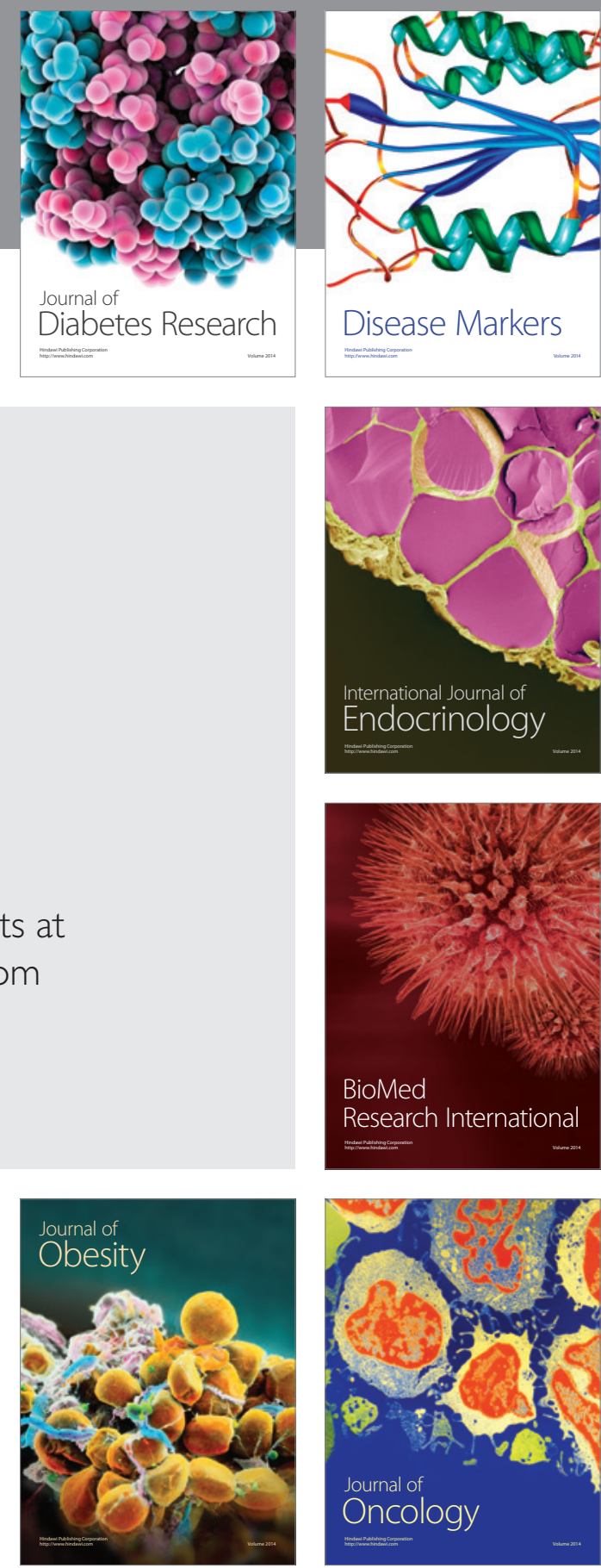

Disease Markers
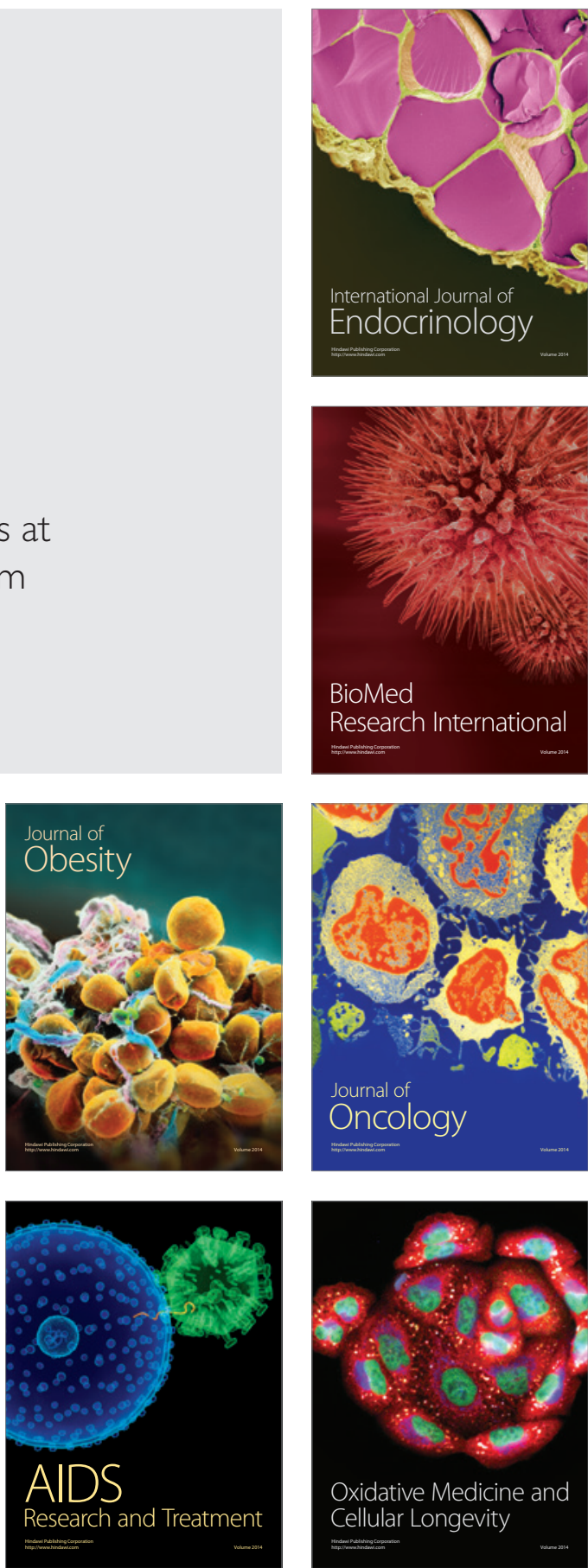